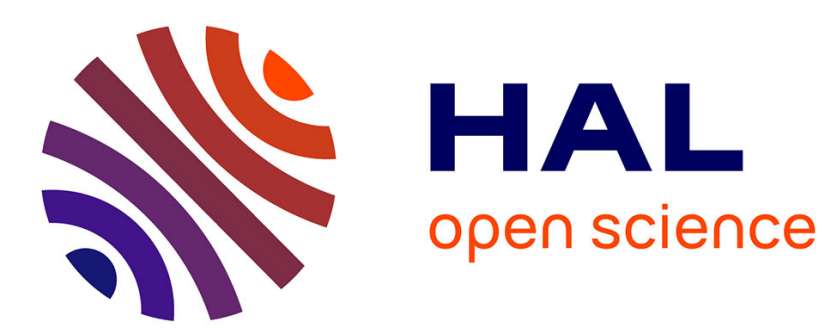

\title{
A stability estimate for the Aleksandrov-Fenchel inequality under regularity assumptions
}

Yves Martinez-Maure

\section{To cite this version:}

Yves Martinez-Maure. A stability estimate for the Aleksandrov-Fenchel inequality under regularity assumptions. 2015. hal-01188332

\section{HAL Id: hal-01188332 \\ https://hal.science/hal-01188332}

Preprint submitted on 28 Aug 2015

HAL is a multi-disciplinary open access archive for the deposit and dissemination of scientific research documents, whether they are published or not. The documents may come from teaching and research institutions in France or abroad, or from public or private research centers.
L'archive ouverte pluridisciplinaire HAL, est destinée au dépôt et à la diffusion de documents scientifiques de niveau recherche, publiés ou non, émanant des établissements d'enseignement et de recherche français ou étrangers, des laboratoires publics ou privés. 


\title{
A stability estimate for the Aleksandrov-Fenchel inequality under regularity assumptions
}

Yves Martinez-Maure

yves.martinez-maure@imj-prg.fr

\begin{abstract}
We give, under appropriate regularity assumptions, a strengthening of the Aleksandrov-Fenchel inequality in the form of a stability estimate.
\end{abstract}

\section{Contents}

1. Introduction and statement of the main result

1.1. The Aleksandrov-Fenchel inequality

1.2. Partial extensions to hedgehogs

1.3. A stability estimate for the Aleksandrov-Fenchel inequality

2. Background on hedgehogs

3. Auxiliary results

4. Proof of Theorem 2

5. Some particular cases

5.1. Planar case

5.2. Bol's inequality

5.3. Case where $L_{3}, \ldots, L_{n+1}$ are the unit ball $B \subset \mathbb{R}^{n+1}$

6. Comparison with a result by Schneider and by Goodey and Groemer

\section{Introduction and statement of the main result}

\subsection{The Aleksandrov-Fenchel inequality}

The classical Aleksandrov-Fenchel inequality

$$
v\left(H, K, L_{3}, \ldots, L_{n+1}\right)^{2} \geq v\left(H, H, L_{3}, \ldots, L_{n+1}\right) v\left(K, K, L_{3}, \ldots, L_{n+1}\right)
$$

MSC 2010: 52A20, 52A30, 52A39, 52A40

Keywords. Hedgehog, Alexandrov-Fenchel inequality, stability estimate 
is a central result in the theory of mixed volumes. Here, $H, K, L_{3}, \ldots, L_{n+1}$ are convex bodies in $(n+1)$-dimensional real Euclidean vector space $\mathbb{R}^{n+1}$ and, $v$ denotes the mixed volume. Many geometric inequalities for convex bodies are consequences of (1) (see, e.g., [12, Chapter 7]). Connections with algebraic geometry have been discovered, which have led to new proofs of (1) via the Hodge index theorem [6, 13]. Equality holds in (1) if $K$ and $L$ are homothetic. However, this is not the only case and until now, the equality problem remains unsolved (see [12, Section 7.6] for a discussion).

Our main result gives, under appropriate regularity assumptions, a strengthening of the Aleksandrov-Fenchel inequality in the form of a stability estimate (see Theorem 2 below). Instead of restricting our attention to convex bodies, we are going to work in the setting of hedgehogs, which can be regarded as the Minkowski differences of arbitrary convex bodies in $\mathbb{R}^{n+1}$ (i.e., as the geometrical realizations of formal differences of convex bodies of $\mathbb{R}^{n+1}$ ). The proof of our main result is based on the study of equality cases in partial extensions of the Aleksandrov-Fenchel inequality to hedgehogs.

\subsection{Partial extensions to hedgehogs}

The set $\mathcal{K}^{n+1}$ of convex bodies of $\mathbb{R}^{n+1}$, equipped with Minkowski addition and multiplication by nonnegative real numbers, forms a commutative semigroup, having the cancellation property, with scalar operator. Of course, it does not constitute a vector space since there is no subtraction in $\mathcal{K}^{n+1}$. Now formal differences of convex bodies of $\mathbb{R}^{n+1}$ form a vector space $\mathcal{H}^{n+1}$ in which $\mathcal{K}^{n+1}$ is a cone that spans the entire space. It is thus natural to consider the multilinear extension of the mixed volume $v:\left(\mathcal{K}^{n+1}\right)^{n+1} \rightarrow \mathbb{R}$ to a symmetric $(n+1)$-linear form on $\mathcal{H}^{n+1}$. We still denote this extension by $v$. Hedgehogs are simply the geometrical realizations of elements of $\mathcal{H}^{n+1}$ in $\mathbb{R}^{n+1}$ (see Section 2 for a short introduction). They are thus the natural geometrical objects when one seeks to extend parts of the Brunn-Minkowski theory to a vector space which contains convex bodies. The idea of considering the multilinear extension of the mixed volume to formal differences of convex bodies may be traced back to papers by A.D. Alexandrov [1] and H. Geppert [4].

Let $\mathbb{S}^{n}$ denote the unit sphere of $\mathbb{R}^{n+1}$. In which follows, we shall identify convex bodies and hedgehogs of $\mathbb{R}^{n+1}$ with their respective support functions. Thus the classical Aleksandrov-Fenchel inequality (1) will be rewritten

$$
v(h, k ; l)^{2} \geq v(h, h ; l) v(k, k ; l)
$$


where $h, k, l_{3}, \ldots, l_{n+1}$ denote the support functions of $H, K, L_{3}, \ldots, L_{n+1}$, $l=\left(l_{3}, \ldots, l_{n+1}\right)$ and $v(f, g ; l):=v\left(f, g, l_{3}, \ldots, l_{n+1}\right)$. We shall see that any real function of class $C^{2}$ on $\mathbb{S}^{n}$ is the support function of some hedgehog in $\mathbb{R}^{n+1}$. In [8], the author gave the following partial extension of the Alexandrov-Fenchel inequality to hedgehogs under the assumption that $l_{3}, \ldots, l_{n+1}$ are of class $C^{2},(n \geq 1)$.

Theorem 1 Let $f: \mathbb{S}^{n} \rightarrow \mathbb{R}$ be a $C^{2}$-function such that $v(f, f ; l)>0$. Then

$$
v(f, g ; l)^{2} \geq v(f, f ; l) v(g, g ; l)
$$

for any $g \in C^{2}\left(\mathbb{S}^{n} ; \mathbb{R}\right)$ and, the equality holds if and only if there exists $(\lambda, \mu) \in \mathbb{R}^{2} \backslash\{(0,0)\}$ such that $\lambda f+\mu g$ be the support function of a point.

\subsection{A stability estimate for the Aleksandrov-Fenchel inequality}

We shall write $k \in C_{+}^{2}\left(\mathbb{S}^{n} ; \mathbb{R}\right)$ to mean that $k$ is the support function of a convex body whose boundary is a hypersurface with positive Gauss curvature. For $\nu \in \mathbb{S}^{n}$, define $\sigma_{\nu}(u):=\frac{1}{2}|\langle u, \nu\rangle|$ for $u \in \mathbb{S}^{n}: \sigma_{\nu}$ is the support function of the unit segment $U(\nu)$ parallel to $\nu$ and centered at the origin. Our main result is the following.

Theorem 2 For $h \in C^{2}\left(\mathbb{S}^{n} ; \mathbb{R}\right), k \in C_{+}^{2}\left(\mathbb{S}^{n} ; \mathbb{R}\right)$ and $l=\left(l_{3}, \ldots, l_{n+1}\right) \in$ $C_{+}^{2}\left(\mathbb{S}^{n} ; \mathbb{R}\right)^{n-1}$

$$
v(h, k ; l)^{2}-v(h, h ; l) v(k, k ; l) \geq \frac{v(k, k ; l)^{2}}{4}\left(M_{(h, k ; l)}-m_{(h, k ; l)}\right)^{2},
$$

where $m_{(h, k ; l)}:=\min _{\nu \in \mathbb{S}^{n}} \frac{v\left(h, \sigma_{\nu} ; l\right)}{v\left(k, \sigma_{\nu} ; l\right)}$ and $M_{(h, k ; l)}:=\max _{\nu \in \mathbb{S}^{n}} \frac{v\left(h, \sigma_{\nu} ; l\right)}{v\left(k, \sigma_{\nu} ; l\right)}$.

Remark. Given $\nu \in \mathbb{S}^{n}$, denote by $\nu^{\perp}$ the vector subspace orthogonal to $\nu$. For any $f \in C^{2}\left(\mathbb{S}^{n} ; \mathbb{R}\right)$, we have

$$
(n+1) v\left(f, \sigma_{\nu} ; l_{3}, \ldots, l_{n+1}\right)=v_{\nu^{\perp}}\left(f^{\nu} ; l_{3}^{\nu}, \ldots, l_{n+1}^{\nu}\right),
$$

where $v_{\nu^{\perp}}$ is the $n$-dimensional mixed volume in $\nu^{\perp}$ and $f^{\nu}, l_{3}^{\nu}, \ldots, l_{n+1}^{\nu}$ the respective restrictions of $f, l_{3}, \ldots, l_{n+1}$ to $\mathbb{S}_{\nu}=\mathbb{S}^{n} \cap \nu^{\perp}$ (see $[9$, Prop. 5]). Remind that if $f \in C^{2}\left(\mathbb{S}^{n} ; \mathbb{R}\right)$ is the support function of a convex body $K$, then $f^{\nu} \in C^{2}\left(\mathbb{S}_{\nu} ; \mathbb{R}\right)$ is the support function of the image of $K$ under orthogonal projection to $\nu^{\perp}$. The notion of mixed projection body extends to hedgehogs (see [9]) and, if we denote by $\Pi_{(f ; l)}$ the mixed projection hedgehog of the hedgehogs with support functions 
$f, l_{3}, \ldots, l_{n+1}$ and by $h_{\Pi_{(f ; l)}}$ its support function, then the inequality of Theorem 2 can be rewritten in the form

$$
v(h, k ; l)^{2}-v(h, h ; l) v(k, k ; l) \geq \frac{v(k, k ; l)^{2}}{4} \mathcal{D}\left(\frac{h_{\Pi_{(h ; l)}}}{h_{\Pi(k ; l)}}\right)^{2},
$$

where $\mathcal{D}\left(\frac{h_{\Pi_{(h ; l)}}}{h_{\Pi(k ; l)}}\right)$ is the diameter of the image of $\mathbb{S}^{n}$ under $\frac{h_{\Pi_{(h ; l)}}}{h_{\Pi(k ; l)}}$.

Our proof is based on the study of equality cases in the extension of Theorem 1 to the case where $f \in C^{2}\left(\mathbb{S}^{n} ; \mathbb{R}\right) \oplus \mathbb{R} \sigma_{\nu}$. It is inspired by the work of G. Bol [2] who proved the result for $n=2$ and $l_{3}=$ 1. Unfortunately, Bol's work has apparently felt into oblivion. This is perhaps due to the fact that Bol's proof contains a series of errors that make it difficult to understand. But fortunately it can be corrected and the approach can be adapted to our more general setting.

\section{Background on hedgehogs}

In this section, we recall for the convenience of the reader the necessary background on hedgehogs.

The set $\mathcal{K}^{n+1}$ of all convex bodies of $(n+1)$-Euclidean vector space $\mathbb{R}^{n+1}$ is usually equipped with Minkowski addition and multiplication by nonnegative real numbers, which are respectively defined by:

(i) $\forall(K, L) \in\left(\mathcal{K}^{n+1}\right)^{2}, K+L=\{u+v \mid u \in K, v \in L\} ;$

(ii) $\forall \lambda \in \mathbb{R}_{+}, \forall K \in \mathcal{K}^{n+1}, \lambda . K=\{\lambda u \mid u \in K\}$.

It does not constitute a vector space since there is no subtraction in $\mathcal{K}^{n+1}$ : not for every pair $(K, L) \in\left(\mathcal{K}^{n+1}\right)^{2}$ does there exist an $X \in \mathcal{K}^{n+1}$ such that $L+X=K$. Now, in the same way as we construct the group $\mathbb{Z}$, of integers from the monoid $\mathbb{N}$ of nonnegative integers, we can construct the vector space $\mathcal{H}^{n+1}$ of formal differences of convex bodies from $\mathcal{K}^{n+1}$. We can then regard $\mathcal{K}^{n+1}$ as a cone of $\mathcal{H}^{n+1}$ that spans the entire space. Hedgehog theory simply consists in:

1. considering each formal difference of convex bodies of $\mathbb{R}^{n+1}$ as a geometrical object in $\mathbb{R}^{n+1}$, called a hedgehog (see below);

2. extending the mixed volume $v:\left(\mathcal{K}^{n+1}\right)^{n+1} \rightarrow \mathbb{R}$ to a symmetric $(n+1)$-linear form on $\mathcal{H}^{n+1}$.

3. extending certain parts of the Brunn-Minkowski theory to $\mathcal{H}^{n+1}$. For $n \leq 2$, it goes back to a paper by H. Geppert [4] who introduced hedgehogs under the German names stützbare Bereiche $(n=1)$ and stützbare Flächen $(n=2)$. 
$\mathbf{C}^{2}$ case. Here we follow more or less [7]. As is well-known, every convex body $K \subset \mathbb{R}^{n+1}$ is determined by its support function $h_{K}: \mathbb{S}^{n} \longrightarrow \mathbb{R}$, where $h_{K}(u)$ is defined by $h_{K}(u)=\sup \{\langle x, u\rangle \mid x \in K\},\left(u \in \mathbb{S}^{n}\right)$, that is, as the signed distance from the origin to the support hyperplane with normal vector $u$. In particular, every closed convex hypersurface of class $C_{+}^{2}$ (i.e., $C^{2}$-hypersurface with positive Gaussian curvature) is determined by its support function $h$ (which must be of class $C^{2}$ on $\mathbb{S}^{n}$ [12, p. 111]) as the envelope $\mathcal{H}_{h}$ of the family of hyperplanes with equation $\langle x, u\rangle=h(u)$. This envelope $\mathcal{H}_{h}$ is described analytically by the following system of equations

$$
\left\{\begin{array}{c}
\langle x, u\rangle=h(u) \\
\langle x, .\rangle=d h_{u}(.)
\end{array} .\right.
$$

The second equation is obtained from the first by performing a partial differentiation with respect to $u$. From the first equation, the orthogonal projection of $x$ onto the line spanned by $u$ is $h(u) u$ and from the second one, the orthogonal projection of $x$ onto $u^{\perp}$ is the gradient of $h$ at $u$ (see Figure 1). Therefore, for each $u \in \mathbb{S}^{n}, x_{h}(u)=h(u) u+(\nabla h)(u)$ is the unique solution of this system.

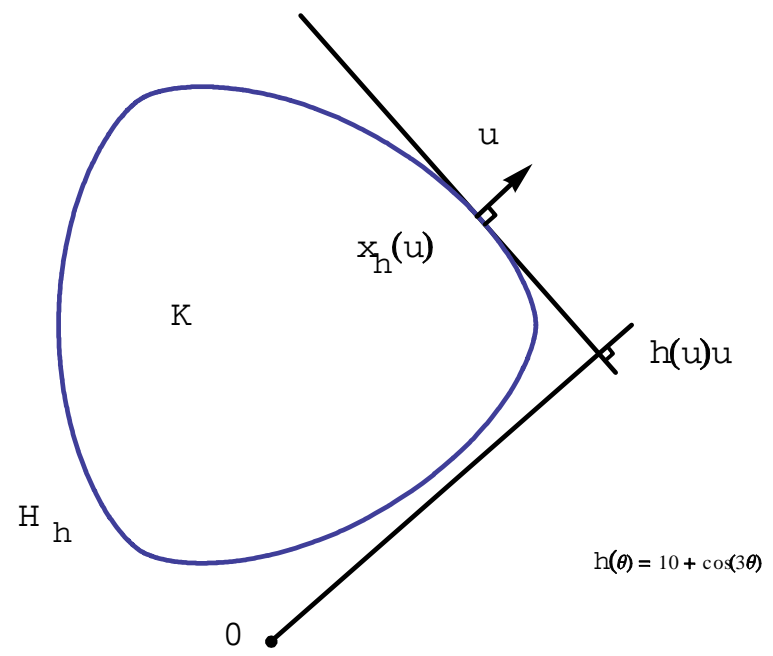

Figure 1. Hedgehogs as envelopes parametrized by their Gauss map

Now, for any $C^{2}$-function $h$ on $\mathbb{S}^{n}$, the envelope $\mathcal{H}_{h}$ is in fact welldefined (even if $h$ is not the support function of a convex hypersurface). Its natural parametrization $x_{h}: \mathbb{S}^{n} \rightarrow \mathcal{H}_{h}, u \mapsto h(u) u+(\nabla h)(u)$ can be 
interpreted as the inverse of its Gauss map, in the sense that: at each regular point $x_{h}(u)$ of $\mathcal{H}_{h}, u$ is a normal vector to $\mathcal{H}_{h}$. We say that $\mathcal{H}_{h}$ is the hedgehog with support function $h$ (cf. Figure 2). Note that $x_{h}$ depends linearly on $h$.

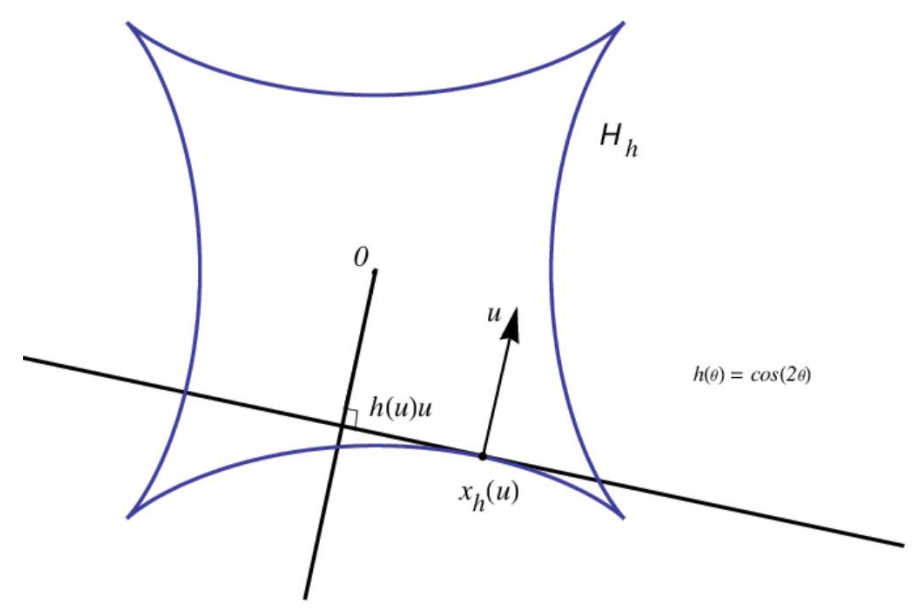

Figure 2. A plane hedgehog with a $C^{2}$-support function

Hedgehogs with a $C^{2}$-support function can be regarded as Minkowski differences of convex hypersurfaces of class $C_{+}^{2}$. Indeed, given any $h \in$ $C^{2}\left(\mathbb{S}^{n} ; \mathbb{R}\right)$, for all large enough real constant $r$, the functions $h+r$ and $r$ are support functions of convex hypersurfaces of class $C_{+}^{2}$ such that $h=(h+r)-r$.

General case. In [10], the author extended the notion of hedgehog by regarding hedgehogs as Minkowski differences of arbitrary convex bodies. The trick is to define hedgehogs inductively as collections of lower-dimensional 'support hedgehogs'. More precisely, the definition of general hedgehogs is based on the three following remarks.

(i) In $\mathbb{R}$, every convex body $K$ is determined by its support function $h_{K}$ as the segment $\left[-h_{K}(-1), h_{K}(1)\right]$, where $-h_{K}(-1) \leq h_{K}(1)$, so that the difference $K-L$ of two convex bodies $K, L$ can be defined as an oriented segment of $\mathbb{R}: K-L:=\left[-\left(h_{K}-h_{L}\right)(-1),\left(h_{K}-h_{L}\right)(1)\right]$.

(ii) If $K$ and $L$ are two convex bodies of $\mathbb{R}^{n+1}$ then for all $u \in \mathbb{S}^{n}$, their support sets with unit normal $u$, say $K_{u}$ and $L_{u}$, can be identified with convex bodies $K_{u}$ and $L_{u}$ of the $n$-dimensional Euclidean vector space $u^{\perp} \simeq \mathbb{R}^{n}$.

(iii) Addition of two convex bodies $K, L \subset \mathbb{R}^{n+1}$ corresponds to that of their support sets with same unit normal vector: $(K+L)_{u}=K_{u}+L_{u}$ 
for all $u \in \mathbb{S}^{n}$; therefore, the difference $K-L$ of two convex bodies $K, L \subset \mathbb{R}^{n+1}$ must be define in such a way that $(K-L)_{u}=K_{u}-L_{u}$ for all $u \in \mathbb{S}^{n}$.

A natural way of defining geometrically general hedgehogs as differences of arbitrary convex bodies is therefore to proceed by induction on the dimension by extending the notion of support set with normal vector $u$ to a notion of support hedgehog with normal vector $u$. Let us give an example in $\mathbb{R}^{2}$. Let $K$ and $L$ be the convex bodies of $\mathbb{R}^{2}$ with support function $h_{K}(x)=\left|\left\langle x, e_{1}\right\rangle\right|+\left|\left\langle x, e_{2}\right\rangle\right|$ and $h_{L}(x)=\left|\left\langle x, e_{3}\right\rangle\right|+\left|\left\langle x, e_{4}\right\rangle\right|$, where $\langle.,$.$\rangle is the standard inner product on \mathbb{R}^{2},\left(e_{1}, e_{2}\right)$ the canonical basis of $\mathbb{R}^{2}$ and $e_{3}, e_{4} \in \mathbb{R}^{2}$ the unit vectors given by $e_{3}=\frac{1}{\sqrt{2}}\left(e_{1}+e_{2}\right)$ and $e_{4}=\frac{1}{\sqrt{2}}\left(e_{1}-e_{2}\right)$. These convex bodies are two squares whose formal difference $K-L$ can be realized geometrically as the hedgehog with support function $h=h_{K}-h_{L}$, which is a regular octagram constructed by connecting every third consecutive vertex of a regular octogon (i.e., a regular star polygon with Schläfli symbol $\{8 / 3\})$ : see Figure 3.
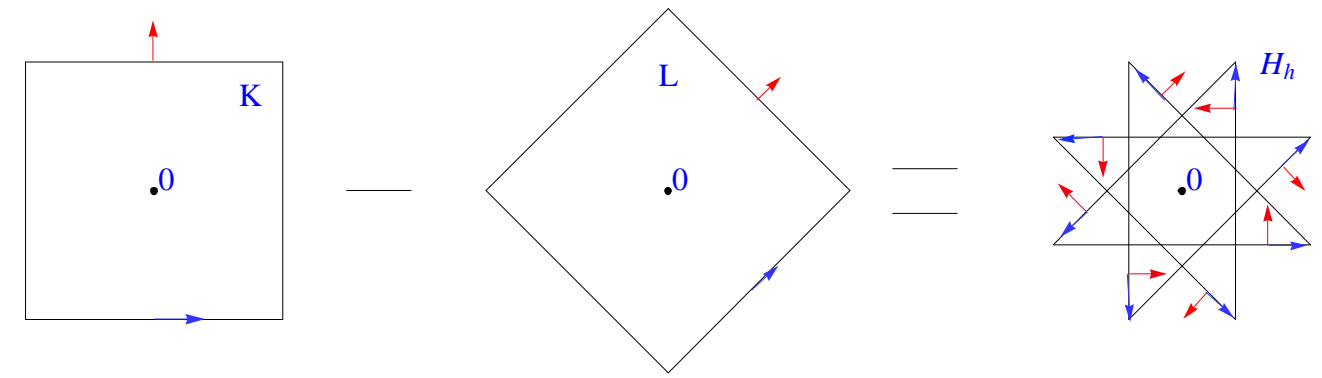

Figure 3. Octagram obtained as the difference of two squares

\section{Gaussian curvature and algebraic volume of $C^{2}$-hedgehogs}

As we saw before, $C^{2}$-hedgehogs (i.e., hedgehogs with a $C^{2}$ support function) may be singular hypersurfaces. Let $\mathcal{H}_{h}$ be such a hedgehog in $\mathbb{R}^{n+1}$. Since the parametrization $x_{h}$ can be regarded as the inverse of the Gauss map, the Gaussian curvature $K_{h}$ of $\mathcal{H}_{h}$ at $x_{h}(u)$ is given by $K_{h}(u)=1 / \operatorname{det}\left[T_{u} x_{h}\right]$, where $T_{u} x_{h}$ is the tangent map of $x_{h}$ at $u$. Therefore, singularities are the very points at which the Gaussian curvature is infinite. For every $u \in \mathbb{S}^{n}$, the tangent map of $x_{h}$ at the point $u$ is $T_{u} x_{h}=h(u) I d_{T_{u} \mathbb{S}^{n}}+H_{h}(u)$, where $H_{h}(u)$ is the symmetric endomorphism associated with the Hessian of $h$ at $u$. Consequently, if $\lambda$ is an eigenvalue of the Hessian of $h$ at $u$ then $\lambda+h(u)$ is (up to the sign) 
one of the principal radii of curvature of $\mathcal{H}_{h}$ at $x_{h}(u)$ and the so-called 'curvature function' $R_{h}:=1 / K_{h}$ can be given by

$$
R_{h}(u)=\operatorname{det}\left[\nabla_{i j} h(u)+h(u) \delta_{i j}\right],
$$

where $\delta_{i j}$ are the Kronecker symbols and $\left(\nabla_{i j} h(u)\right)$ the Hessian of $h$ at $u$ with respect to an orthonormal frame on $\mathbb{S}^{n}$.

The index of a point $x \in \mathbb{R}^{n+1} \backslash \mathcal{H}_{h}$ with respect to $\mathcal{H}_{h}$, say $i_{h}(x)$, can be defined as the degree of the map

$$
\mathcal{U}_{(h, x)}: \mathbb{S}^{n} \rightarrow \mathbb{S}^{n}, u \longmapsto \frac{x_{h}(u)-x}{\left\|x_{h}(u)-x\right\|},
$$

and interpreted as the algebraic intersection number of an oriented halfline with origin $x$ with the hypersurface $\mathcal{H}_{h}$ equipped with its transverse orientation (number independent of the oriented half-line for an open dense set of directions) [7]. For $n+1=2$, the index $i_{h}(x)$ is nothing but the winding number of $\mathcal{H}_{h}$ around $x$ : it counts the total number of times that $\mathcal{H}_{h}$ winds around $x$. For instance, the index is equal to -1 at any interior point of the hedgehog represented on Figure 2, since the curve winds once clockwise around the point.

The (algebraic $(n+1)$-dimensional) volume of $\mathcal{H}_{h}$ can be defined by

$$
v_{n+1}(h):=\int_{\mathbb{R}^{n+1} \backslash \mathcal{H}_{h}} i_{h}(x) d \lambda(x),
$$

where $\lambda$ denotes the Lebesgue measure on $\mathbb{R}^{n+1}$, and it satisfies

$$
v_{n+1}(h)=v(h, \ldots, h)=\frac{1}{n+1} \int_{\mathbb{S}^{n}} h(u) R_{h}(u) d \omega(u),
$$

where $R_{h}$ is the curvature function and $\omega$ the spherical Lebesgue measure on $\mathbb{S}^{n}[7]$. For instance, in the example of Figure 2, the algebraic area (or 2-dimensional volume) of the plane hedgehog $\mathcal{H}_{h}$ is equal to minus the area of the interior of the curve.

\section{Auxiliary results}

First, we fix $\nu \in \mathbb{S}^{n}$ and extend Theorem 1 by replacing $C^{2}\left(\mathbb{S}^{n} ; \mathbb{R}\right)$ by the real vector space, say $V(\nu)$, spanned by $C^{2}\left(\mathbb{S}^{n} ; \mathbb{R}\right)$ and $\sigma_{\nu}$ in $C\left(\mathbb{S}^{n} ; \mathbb{R}\right)$.

Theorem 3 Let $f$ be a function in $V(\nu)$ such that $v(f, f ; l)>0$. Then

$$
v(f, g ; l)^{2} \geq v(f, f ; l) v(g, g ; l)
$$

for all $g \in V(\nu)$ and, the equality holds if and only if there exists $(\lambda, \mu) \in \mathbb{R}^{2} \backslash\{(0,0)\}$ such that $\lambda f+\mu g$ is the support function of a point. 
Proof of Theorem 3. Let $q: V(\nu) \rightarrow \mathbb{R}$ be the quadratic form given by $q(h):=v(h, h ; l)$. Denote by $b$ its polar form: $b(h, k):=v(h, k ; l)$ for $(h, k) \in V(\nu)^{2}$. We start with an observation concerning the restriction of $q$ to the linear subspace $F(\nu)$ of $V(\nu)$ with equation $b(1, h)=0$.

Lemma. If $h$ is in $F(\nu)$ and is not the support function of a point, then $q(h):=v(h, h ; l)<0$.

Proof of Lemma. Such a function $h$ can be decomposed as $h=\gamma+\lambda \sigma_{\nu}$, where $\gamma \in C^{2}\left(\mathbb{S}^{n} ; \mathbb{R}\right)$ and $\lambda \in \mathbb{R}$. From Theorem 1 , we may assume that $\lambda \neq 0$. Replacing $h$ by $-h$ if necessary, we may assume that $\lambda>0$. Choose a number $\varepsilon>0$ small enough so that $1+\varepsilon h$ is the support function of a convex body. Such a number exists by Theorems 1.5.13 and 1.7.1 from [12]. Now, by Theorem 7.6.8 from [12], we know that equality holds in the classical Aleksandrov-Fenchel inequality (1) if and only if $H$ and $K$ are homothetic provided that $L_{3}, \ldots, L_{n+1}$ are smooth convex bodies. So, with our choice of $\varepsilon$, we must have $b(1,1+\varepsilon h)^{2}>q(1) q(h)$.

If $q(h)$ was nonnegative, then the quadratic form $q$ would be positive semi-definite on the linear subspace $V_{h}$ of $V(\nu)$ spanned by 1 and $h$ so that we should have

$$
b(\alpha, \beta)^{2} \leq q(\alpha) q(\beta) \quad \text { for all }(\alpha, \beta) \in V_{h}^{2}
$$

by the Cauchy-Schwarz inequality, which is contradictory.

End of the proof of Theorem 3. Let $P$ be the degree 2 polynomial function given by

$$
P(t):=q(g+t f)=q(g)+2 t b(f, g)+t^{2} q(f) \text { for } t \in \mathbb{R} .
$$

Since $q(f)>0, P(t)>0$ for all large enough $t$. Furthermore, the lemma ensures that $b(1, f) \neq 0$ so that we may define

$$
\tau:=-\frac{b(1, g)}{b(1, f)}
$$

and consider $g+\tau f$, which belongs to $F(\nu)$. Thus, by the lemma, $P(\tau)<0$ unless $g+\tau f$ is the support function of a point. By considering the discriminant of $P$, we deduce that $b(f, g)^{2}>q(f) q(g)$ unless $g+\tau f$ is the support function of a point. Finally, note that if there exists $(\lambda, \mu) \in \mathbb{R}^{2} \backslash\{(0,0)\}$ such that $\lambda f+\mu g$ is the support function of a point, then $b(f, g)^{2}=q(f) q(g)$.

Next, we deduce the following. 
Theorem 4 Let $f$ be a function in $V(\nu)$ such that $v(f, f ; l)>0$. If $g$ is any function in $V(\nu)$ such that $v(f, g ; l)=v(g, g ; l)=0$, then the hedgehog $\mathcal{H}_{g}$ is reduced to a point.

In other words:

Theorem 5 Let $f \in V(\nu)$. If there exists a hedgehog not reduced to a point with support function $g \in V(\nu)$ such that $v(f, g ; l)=v(g, g ; l)=0$, then $v(f, f ; l) \leq 0$.

Proof of Theorem 4. It follows from assumptions that we are in an equality case of (2). So, by Theorem 3 , there exists $(\lambda, \mu) \in \mathbb{R}^{2} \backslash\{(0,0)\}$ such that $\lambda f+\mu g$ is the support function of a point. Since $\mathcal{H}_{\lambda f+\mu g}$ is a point, $v(\lambda f+\mu g, \lambda f+\mu g ; l)=0$. Developing by multilinearity and using assumptions, we deduce that $\lambda^{2} v(f, f ; l)=0$. Since $v(f, f ; l)>0$, $\lambda=0$ and hence $\mathcal{H}_{\mu g}$ is reduced to a point. Now $\mu \neq 0$ since $(\lambda, \mu) \neq$ $(0,0)$. Therefore, $\mathcal{H}_{g}$ is reduced to a point.

\section{Proof of Theorem 2}

Finally, we apply Theorem 5 to

$$
g:=\sigma_{\nu} \quad \text { and } \quad f:=h-\lambda k, \text { where } \quad \lambda:=\frac{v\left(h, \sigma_{\nu} ; l\right)}{v\left(k, \sigma_{\nu} ; l\right)} .
$$

Let us check that all the assumptions of Theorem 5 are then satisfied. Of course, $\mathcal{H}_{g}$ is not reduced to a point since it is a unit segment $U(\nu)$. Since the mixed volume $v: V(\nu)^{n+1} \rightarrow \mathbb{R}$ is linear in each of its arguments, we have $v(f, g ; l)=v\left(h, \sigma_{\nu} ; l\right)-\lambda v\left(k, \sigma_{\nu} ; l\right)=0$. Applying formula (5.77) from [12, p. 302], we obtain

$$
(n+1) v\left(\sigma_{\nu}, \sigma_{\nu} ; l\right)=v_{\nu \perp}\left(U(\nu)^{\nu}, L_{3}^{\nu}, \ldots, L_{n+1}^{\nu}\right),
$$

where $v_{\nu^{\perp}}$ denotes the $n$-dimensional mixed volume in the linear subspace orthogonal to $\nu$ and, $U(\nu)^{\nu}, L_{3}^{\nu}, \ldots, L_{n+1}^{\nu}$ the respective images of $U(\nu), L_{3}, \ldots, L_{n+1}$ under orthogonal projection to this subspace, and thus $v(g, g ; l)=0$ since $U(\nu)^{\nu}=\{0\}$.

Hence by Theorem 5 , we have

$$
v(h-\lambda k, h-\lambda k ; l) \leq 0 .
$$

After replacing $\lambda$ by its value and rearranging, we obtain

$$
v(h, k ; l)^{2}-v(h, h ; l) v(k, k ; l) \geq\left(v(h, k ; l)-\frac{v\left(h, \sigma_{\nu} ; l\right)}{v\left(k, \sigma_{\nu} ; l\right)} v(k, k ; l)\right)^{2}
$$


Using the inequality $a^{2}+b^{2} \geq \frac{1}{2}(a-b)^{2}$ with $a:=v(h, k ; l)-$ $m_{(h, k ; l)} v(k, k ; l)$ and $b:=v(h, k ; l)-M_{(h, k ; l)} v(k, k ; l)$, we deduce that

$$
v(h, k ; l)^{2}-v(h, h ; l) v(k, k ; l) \geq \frac{v(k, k ; l)^{2}}{4}\left(M_{(h, k ; l)}-m_{(h, k ; l)}\right)^{2} .
$$

\section{Some particular cases}

\subsection{Planar case}

The proof of Theorem 2 also works for $n=1$ (i.e., without the $l$ term). If for any hedgehog $\mathcal{H}_{f}$ of $\mathbb{R}^{n+1}$, we define the width of $\mathcal{H}_{f}$ in the direction $\nu \in \mathbb{S}^{n}$ as the signed distance between the support lines of $\mathcal{H}_{f}$ with unit normal $-\nu$ and $\nu$, that is by $w(f, \nu):=f(\nu)-(-f(-\nu))=f(\nu)+$ $f(-\nu)$, then we have:

Corollary 6 For $h \in C^{2}\left(\mathbb{S}^{1} ; \mathbb{R}\right)$ and $k \in C_{+}^{2}\left(\mathbb{S}^{1} ; \mathbb{R}\right)$,

$$
\begin{array}{r}
v(h, k)^{2}-v(h, h) v(k, k) \geq \frac{v(k, k)^{2}}{4}\left(M_{(h, k)}-m_{(h, k)}\right)^{2}, \\
\text { where } m_{(h, k)}:=\min _{\nu \in \mathbb{S}^{1}} \frac{w(h, \nu)}{w(k, \nu)} \text { and } M_{(h, k)}:=\max _{\nu \in \mathbb{S}^{1}} \frac{w(h, \nu)}{w(k, \nu)} .
\end{array}
$$

For $k=1$, this gives :

Corollary 7 For $h \in C^{2}\left(\mathbb{S}^{1} ; \mathbb{R}\right)$,

$$
l(h)^{2}-4 \pi a(h) \geq \frac{\pi^{2}}{4}(D(h)-d(h))^{2},
$$

where $l(h):=2 v(1, h)$ and $a(h):=v(h, h)$ are respectively the (algebraic) length and area of the plane hedgehog $\mathcal{H}_{h}$, and, where $d(h):=$ $\min _{\nu \in \mathbb{S}^{1}} w(h, \nu)$ and $D(h):=\max _{\nu \in \mathbb{S}^{1}} w(h, \nu)$.

Note that $l(h)$ is the length of the plane hedgehog $\mathcal{H}_{h}$ counted with the sign of its curvature function $R_{h}$. If $\mathcal{H}_{h}$ is convex, we have

$$
2 r(h) \leq d(h) \leq D(h) \leq 2 R(h),
$$

where $r(h)$ and $R(h)$ denote respectively the inradius and circumradius of $\mathcal{H}_{h}$. In this particular case, Bonnesen's inequality (see [12, p. 388] and references herein)

$$
l(h)^{2}-4 \pi a(h) \geq \pi^{2}(R(h)-r(h))^{2}
$$

is therefore better. But Corollary 7 remains true without any convexity assumption. 


\subsection{Bol's inequality}

Let $B$ denote the unit ball of $\mathbb{R}^{n+1}$. For $n=2$ and $k=l_{3}=1$, we retrieve Bol's inequality

$$
M^{2}-4 \pi S \geq(L-l)^{2},
$$

for a convex body $K$ of class $C_{+}^{2}$ in $\mathbb{R}^{3}$, where $M$ is the integral of mean curvature $3 v(K, B, B), S$ the surface area $3 v(K, K, B)$ and, $l$ and $L$ the least and greatest perimeter of an orthogonal plane projection [2]. In fact, the result is more general since it remains true for any $C^{2}$-hedgehog $\mathcal{H}_{h}$ of $\mathbb{R}^{3}$ (replacing volumes, areas and lengths by their algebraic versions).

Recall that the girth of $K$ in the direction $\nu \in \mathbb{S}^{2}$ is defined as the perimeter of the orthogonal projection of $K$ onto the linear plane that is orthogonal to $\nu$. Note that the right-hand side of Bol's inequality vanishes if, and only if, $K$ is of constant girth. Since the orthogonal projections of a convex body of constant width are again convex bodies of the same constant width and since, according Barbier's theorem, a planar convex body of constant width $w$ has perimeter $\pi w$, it is clear that every three-dimensional convex body of constant width is of constant girth. Now, H. Minkowski proved in 1904 that the converse is true: every three-dimensional convex body of constant girth is also of constant width: see for instance [3, p. 430]. Therefore, the right-hand side of Bol's inequality vanishes if, and only if, $K$ is of constant width.

\subsection{Case where $L_{3}, \ldots, L_{n+1}$ are the unit ball $B$ of $\mathbb{R}^{n+1}$}

If in Theorem $2, l_{3}, \ldots, l_{n+1}$ are the support functions of the Euclidean unit ball $B$ (that is, if $l_{3}=\ldots=l_{n+1}=1$ ), then, denoting by 1 the $(n-2)$-tuple $(1, \ldots, 1)$, we have:

Corollary 8 For $h \in C^{2}\left(\mathbb{S}^{n} ; \mathbb{R}\right)$ and $k \in C_{+}^{2}\left(\mathbb{S}^{n} ; \mathbb{R}\right)$,

$$
v_{11}^{2}-v_{20} v_{02} \geq \frac{1}{4}\left(M_{(h, k)}-m_{(h, k)}\right)^{2} v_{02}^{2},
$$

where $v_{i j}:=v(h[i], k[j] ; \mathbf{1}), h[i]$ meaning that $h$ appears $i$ times and

$k[j]$ that $k$ appears $j$ times, and, where $m_{(h, k)}:=\min _{\nu \in \mathbb{S}^{n}} \frac{v\left(h, \sigma_{\nu} ; \mathbf{1}\right)}{v(k, \sigma ; \mathbf{1})}$ and $M_{(h, k)}:=\max _{\nu \in \mathbb{S}^{n}} \frac{v\left(h, \sigma_{\nu} ; \mathbf{1}\right)}{v\left(k, \sigma_{\nu} ; \mathbf{1}\right)}$. 


\section{Comparison with a result by Schneider and by Goodey and Groemer}

With each convex body $K$ of positive dimension in $\mathbb{R}^{n+1}$, is associated its normalized homothetic copy $\bar{K}$ defined by

$$
\bar{K}:=\frac{K-s(K)}{w(K)}
$$

(see [12, p. 421]). The following theorem, proved by R. Schneider [11] and independently by P. R. Goodey and H. Groemer [5], may be interpreted as a stability result in a specific case of the Alelsandrov-Fenchel inequality.

Theorem (Schneider, Goodey-Groemer). If $H$ and $K$ are two convex bodies of positive dimension in $\mathbb{R}^{n+1}$, then

$$
v_{11}^{2}-v_{20} v_{02} \geq \frac{n+2}{n(n+1)} w(H)^{2} \delta_{2}(\bar{H}, \bar{K})^{2} v_{02},
$$

where $w(H)$ is the mean width of $H, \bar{H}$ and $\bar{K}$ the respective normalized homothetic copies of $H$ and $K, v_{i j}$ the mixed volume of $i$ copies of $H$ with $j$ copies of $K$ and $n-1$ copies of the unit ball $B$, and

$$
\delta_{2}(\bar{H}, \bar{K})^{2}:=\int_{\mathbb{S}^{n}}\left(h_{\bar{H}}-h_{\bar{K}}\right)^{2} d S,
$$

denoting by $S$ the surface measure on the sphere $\mathbb{S}^{n}$ and by $h_{L}$ the support function of a convex body $L$.

This stability result has to be compared to Corollary 8 (and thus to Corollary 7 in the planar case). Note that the assumptions overlap only partially: in Corollary $8, h$ and $k$ have to be $C^{2}$ but $\mathcal{H}_{h}$ is not necessarily convex. When we take the union of assumptions of the results under consideration, the above theorem is better in some cases (take for instance $K=B$ and $H$ of constant width in $\mathbb{R}^{3}$ ) while particular cases of Corollary 8 are better in other cases (take for instance $k(\theta):=1$ and $\left.h(\theta):=1+\frac{1}{4} \sin t \cos ^{7} t,\left(\theta \in \mathbb{S}^{1}=\mathbb{R} / 2 \pi \mathbb{Z}\right)\right)$.

\section{References}

[1] A. D. Aleksandrov, Zur Theorie der gemischten Volumina von konvexen Körpern, I: Verallgemeinerung einiger Begriffe der Theorie der konvexen Körper (in Russian). Mat. Sbornik N. S. 2 (1937), 947-972. 
[2] G. Bol, Zur Theorie der konvexen Körper. Jahresber. Deutsche Math.-Ver. 49 (1939),113-123.

[3] R. J. Gardner, Geometric Tomography. Cambridge Univ. Press, New York, 1995.

[4] H. Geppert, Über den Brunn-Minkowskischen Satz, Math. Z. 42 (1937), 238-254.

[5] P.R. Goodey and H. Groemer, Stability results for first order projection bodies. Proc. Am. Math. Soc. 109 (1990), 1103-1114.

[6] A.G. Khovanskiı, Newton polyhedra and genus of complete intersections. Funktsional. Anal. i Prilozhen. 12 (1978) 51-61, Funct. Anal. Appl. 12 (1978) 38-46.

[7] R. Langevin, G. Levitt and H. Rosenberg, Hérissons et multihérissons (enveloppes paramétrées par leur application de Gauss). In: Singularities, Banach Center Publ. 20, Warsaw, PWN, 1988, 245-253.

[8] Y. Martinez-Maure, De nouvelles inégalités géométriques pour les hérissons. Arch. Math. 72 (1999), 444-453.

[9] Y. Martinez-Maure, Hedgehogs and zonoids. Adv. Math. 158 (2001), 1-17.

[10] Y. Martinez-Maure, Théorie des hérissons et polytopes. C. R. Acad. Sci. Paris, Sér. I, 336 (2003), 241-244.

[11] R. Schneider, Stability in the Aleksandrov-Fenchel-Jessen theorem. Mathematika 36 (1989), 50-59

[12] R. Schneider, Convex Bodies: The Brunn-Minkowski Theory, 2nd expanded ed., Cambridge Univ. Press, 2014.

[13] B. Teissier, Du théorème de l'index de Hodge aux inégalités isopérimétriques, C. R. Acad. Sci. Paris Sér. A-B 288 (1979) 287289.

Y. Martinez-Maure

Institut Mathématique de Jussieu - Paris Rive Gauche

UMR 7586 du CNRS

Bâtiment Sophie Germain

Case 7012

75205 Paris Cedex 13

France 